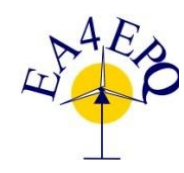

$19^{\text {th }}$ International Conference on Renewable Energies and Power Quality (ICREPQ’21)

Almeria (Spain), $28^{\text {th }}$ to $30^{\text {th }}$ July 2021

Renewable énergy and Power Quality fournal (RE\&PQJ)

ISSN 2172-038 X, Volume No.19, September 2021

\title{
Synthetic Series of Electricity Generation through a Photovoltaic System by using Different Panel Temperature Models
}

\author{
A. H. M. Stach ${ }^{1,2,3}$, D. P. Neto ${ }^{1,2,3}$, M. A. Vidal ${ }^{2,3}$, O. C. N. Souto 2 , S. B. Silva ${ }^{2}$ and E. G. Domingues ${ }^{1,2,3}$ \\ Master's Program in Sustainable Process Technology ${ }^{1}$ \\ Electrical Engineering/Control and Automation Engineering Program ${ }^{2}$ \\ NeXT - Nucleus of Experimental and Technological Studies ${ }^{3}$ \\ Federal Institute of Education, Science, and Technology of Goiás, Brazil \\ Rua 75, nº 46 - Centro, Goiânia - GO, 74055-110 (Brasil) \\ E-mail: aironherbertms@gmail.com, prof.eldergd@gmail.com
}

\begin{abstract}
The electric energy produced by photovoltaic systems is a function of several variables that present random behavior, such as solar irradiance, ambient temperature, wind speed, and relative humidity. Several models describe the energy produced by these systems, which reflect the various factors that influence the generation and how significant these influences are. In this way, the present work aims to compare some panel temperature models and the electrical energy produced from a photovoltaic system located in the Midwest of Brazil. The applied methodology aims to evaluate the expected averages and standard deviations of the monthly synthetic series of generated energy to identify the main random variables that influence the electric generation. The results indicate the model that best describes the physical system concerning the randomness of the variables involved, the photovoltaic technology, the location, and the measurement period.
\end{abstract}

Keywords. Stochastic Modeling, Photovoltaic Systems, Panel Temperature, Synthetic Series

\section{Introduction}

Brazil has a high potential to generate electricity from hydroelectric power plants. However, despite being a renewable source, the implementation of hydroelectric dams is linked to many problems that make them unsustainable, such as changes in natural waterways, changes in riverine populations, loss of biodiversity, greenhouse gas emissions in the organic decomposition of the reservoir, among others [1].

In this scenario, it is important to diversify the Brazilian energy matrix, which is predominantly hydro, in sources with lower environmental impacts, such as photovoltaic. Photovoltaic solar energy is the fastest-growing energy source in the world, with an increase of $31.2 \%$ from 2017 to 2018 , while wind energy was second with an increase of $12.2 \%$ during the same period [2].

The electric current produced by photovoltaic panels depends on the intensity of the solar radiation that hits the cells. Additionally, the electrical voltage produced by the panels is influenced by their operating temperature. In other words, the electrical energy supplied by photovoltaic panels is a function of the solar irradiance and the module temperature [3].

Most studies on photovoltaic systems analyze the technical and economic viability of small-scale power plants, aimed at self-production in homes and businesses in a deterministic manner [4], [5]. Other studies focus on policy strategies to encourage the growth of renewable generation sources [6].

Lourenço et al. [7] compare several stochastic models for the generation of solar irradiance synthetic series with historical data. The authors make use of the autoregressive model, the autoregressive integrated model, the autoregressive moving average model, and the autoregressive integrated moving average model. The results show that the autoregressive integrated model applied to monthly averages is more suitable for the generation of synthetic irradiance series in northern Brazil.

Some works compare various photovoltaic panel temperature models with measurement values using deterministic techniques [8], [9], [10]. The authors calculate the errors between the data, disregarding the uncertainties of the random variables, and identify the best models for panel temperature prediction under the applied conditions.

There is great difficulty in predicting the energy generated by photovoltaic systems over the project life. This is mainly because of the panel temperature and the power output which depend on several stochastic factors, such as solar irradiance, ambient temperature, wind speed, humidity, and wind direction.

The volume of studies produced, especially for stochastic analysis, in renewable plants in Brazil is summarized. Most studies that use stochastic methods for renewable energy sources focus on economics risk analysis. Pereira et al. [11] present a risk analysis methodology, using commercial software to apply the Monte Carlo Method (MCM), to evaluate the economic viability of a solar photovoltaic plant installed in the state of Pará, Brazil. Pinheiro Neto et al. [12] present methodology for risk analysis of investment in a 
solar photovoltaic plant in Brazil. The authors use the MCM under the Free Contracting Environment and consider the physical variables solar irradiance and ambient temperature for the panel temperature calculation.

In this context, this study aims to fill the gap in the small number of studies that consider the random variables to estimate the electricity generated from photovoltaic systems during the project lifetime.

\section{Methodology}

Fig. 1 presents the flowchart of the methodology used in this work. The following steps will be described individually.

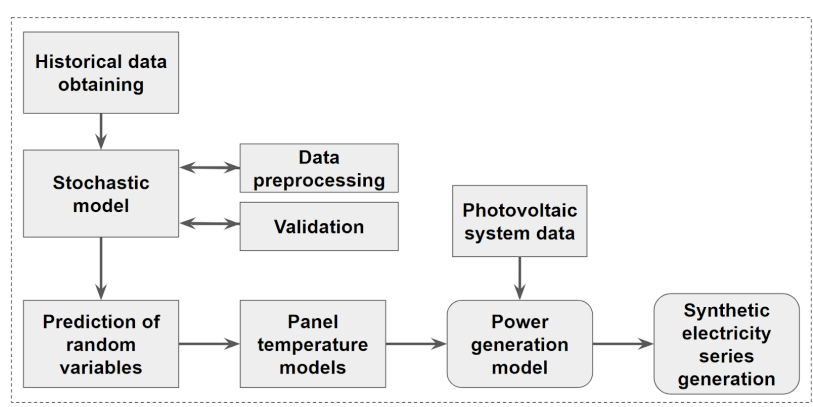

Fig. 1. Flowchart of the methodology

\section{A. Historical Data Obtaining}

The database was the National Solar Radiation Database (NSRDB) from National Renewable Energy Laboratory (NREL). This base is a collection of semi-hourly values of several climatic variables, such as solar irradiance $(I)$, ambient temperature $\left(T_{a}\right)$, wind speed $(W)$, relative humidity $(H)$, wind direction $\left(W_{d i r}\right)$, and others. Data obtained from NSRDB uses a physical solar model, and its coverage is limited by latitudes from $-20^{\circ}$ to $60^{\circ}$ and longitudes from $-25^{\circ}$ to $175^{\circ}$.

Historical data from NSRDB were requested on the NREL website and later received in the informed e-mail. The latitude and the longitude coordinates of the locality, the period of interest, and the desired variables are selected. The data are exported in annual spreadsheets, with measurements every 30 minutes in the chosen period for all selected variables.

The historical data processing and all the rest of the calculations were performed in Matlab, which is a high-performance interactive software used by engineers and scientists for computational and mathematical purposes. After inserting the spreadsheets in the computational tool, the monthly averages are calculated for each variable in the chosen period.

\section{B. Stochastic Model}

After obtaining the historical data of the random variables, stochastic models were built, which will serve to obtain the prediction of these variables over the project lifetime.
Several models have been tested to describe the behavior of the random variables, such as the Autoregressive (AR) with different orders and lags, Moving Average (MA), and Autoregressive Moving Average (ARMA). The model that best fit the historical data and that was used in this work was the AR model with one term and order estimated by the autocorrelation function of the mensal data. This model made it possible to obtain monthly synthetic series with averages and standard deviations closest to the monthly historical data.

Autoregressive (AR) models can be used in time series modeling, where the current value depends on past values plus a constant and a random error (white noise). You can build the AR model from the following equation [13], [14], [15]:

$$
z_{i}=a_{1} z_{i-1}+a_{2} z_{i-2}+\ldots+a_{k} z_{i-k}+c+e_{i}
$$

Where $z_{i}$ is the autoregressive process of order $k, e_{i}$ is the white noise of instant $i, a_{k}$ is the estimated coefficient of process $\mathrm{AR}$ and $\mathrm{c}$ is a constant.

White Gaussian noise or random error is a sequence of random numbers without any autocorrelation, with zero mean and constant estimated variance. For the generation of the synthetic series, sequences of random numbers of the same duration of the project life are used for each generated scenario.

\section{Data Preprocessing}

To estimate the parameters of the adopted AR model, it is necessary to preprocess the data, which consists of removing trends (seasonality) from the monthly averages of the historical data set. Trend removal has to be executed because the structure of the AR model is specific to represent stochastic processes that have zero mean [14]. After the synthetic series generation process, the trends are reinserted in the series for the adequate representation of the random variable. To obtain the trends, (2) is used. To remove the trend from the historical series, (3) is applied [14], [15]:

$$
\begin{gathered}
t_{i}=\frac{1}{N} \sum_{j=1}^{N} x(i, j) \\
x^{\prime}(i, j)=\frac{x(i, j)}{t_{i}}-1
\end{gathered}
$$

Where $t_{i}$ is the trend of month $i, N$ is the total number of annual series, $x(i, j)$ is the average solar irradiance value of month $i$ and year $j$, and $x^{\prime}(i, j)$ represents the trendless historical series of the month $i$ and the year $j$.

After data preprocessing, we estimate the coefficients and variance of white noise present in the AR model. For this, a parameter estimation method must be applied. There are several methods for this purpose, such as Yule-Walker [16], 
[17], the least-squares method [18], the maximum likelihood method [19], and others. After applying the stochastic model to generate the synthetic series, it is necessary to reinsert the trends, which can be done from the inverse operation of (3).

\section{Model Validation}

To validate the model with the historical series, the Mean Relative Percentage Error (MPE) was used, which indicates the mean absolute error quotient and the historical value, weighting the individual errors by their representativeness concerning the actual value (the monthly historical data) [13], [20].

The equation used to calculate the MPE is presented below.

$$
M P E=\frac{100}{n} \sum_{j=1}^{n} \frac{\left.y_{j}-\widehat{y}_{j}\right\rfloor}{y_{j}}
$$

Where $n=1, \ldots, 240$ (equivalent to 20 years of history), $y_{j}$ is the value of history and $\hat{y}_{j}$ is the predicted value.

The minimum values, the maximum values, the global averages, and the standard deviations were also used in the model validation stage. The calculation of the minimum and maximum is done individually for each of the scenarios, then the arithmetic average is calculated. The global averages and standard deviations are obtained from the averages of the synthetic series.

\section{E. Prediction of Random Variables}

After validation, the stochastic model obtained is used for the prediction of the random variables (solar irradiance, ambient temperature, wind speed, relative humidity, and wind direction). The monthly synthetic series of the random variables are then used to obtain the monthly synthetic series of the panel temperature and the generated electrical energy.

\section{F. Photovoltaic Panel Temperature Models}

Panel temperature is directly related to ambient temperature, solar irradiance, wind speed, relative humidity, and wind direction [21]. The panel temperature model that considers only solar irradiance and ambient temperature, presented by Masters [22], can be expressed by (5). The panel temperature model that considers solar irradiance, ambient temperature, and wind speed, presented by Tamizhmani et al. [23] and Pinheiro Neto et al. [24], is expressed by (6). The panel temperature model that considers solar irradiance, ambient temperature, wind speed, relative humidity, and wind direction, also presented by Tamizhmani et al. [23], is given by (7).

$$
\begin{gathered}
T_{P A}=T_{a}+0.03125 \cdot I \\
T_{P B}=0.926 \cdot T_{a}+0.030 \cdot I-1,666 \cdot \mathrm{W}+5.1
\end{gathered}
$$

$$
\begin{aligned}
& T_{P C}=0.954 \cdot T_{a}+0.03 \cdot I-1.629 \cdot W \\
& \quad+0.088 \cdot H-0.005 \cdot W_{d i r}+3.9
\end{aligned}
$$

Where $T_{P}$ is the panel temperature calculated from models $\mathrm{A}, \mathrm{B}$, and $\mathrm{C}, T_{a}$ is the ambient temperature in ${ }^{\circ} \mathrm{C}, I$ is the solar irradiance in $\mathrm{W} / \mathrm{m}^{2}, W$ is the wind speed in $\mathrm{m} / \mathrm{s}, H$ is the percent relative humidity, and $W_{d i r}$ is the wind direction angle to the geographic north.

\section{G. Power Generation Model}

The power generated by each panel can be calculated using the Osterwald model [24], expressed by (8).

$$
P_{\text {panel }}=\frac{I}{I_{S T C}} P_{\max _{S T C}}\left[1+\gamma_{T}\left(T_{\text {panel }}-T_{S T C}\right)\right]
$$

Where $I_{S T C}$ is the solar irradiance under standard test conditions, $P_{\max _{S T C}}$ is the maximum power under standard test conditions, $\gamma_{T}$ is the coefficient of variation of power with temperature, $T_{\text {panel }}$ is the panel temperature, $T_{S T C}$ is the temperature under standard test conditions, and $I$ is the solar irradiance on an inclined surface, given by (9).

$$
I=I_{H} \cdot \psi_{m} \cdot F_{c}
$$

Where $I_{H}$ is the solar irradiance on the horizontal surface, $\psi_{m}$ is the factor that converts the incident solar radiation on a horizontal surface to an inclined surface (excluding anisotropic reflections) in the month $m$, and $F_{c}$ is the multiplier factor representing anisotropic reflections, which is a function of the albedo.

The power of the plant is calculated by multiplying the power of the panel from (8), the number of panels, and the Performance Ratio (PR).

\section{H. Synthetic Electricity Series Generation}

The monthly synthetic series of electricity generated by the photovoltaic system is obtained by multiplying the monthly synthetic series of power generated by the standardized number of hours per month (720 hours).

\section{Results}

This study was conducted for a photovoltaic system installed at the Federal Institute of Goiás (IFG), the campus of Itumbiara city, located in the Midwest region of Brazil. All historical data of the random variables (irradiance, ambient temperature, wind speed, relative humidity, and wind direction) were obtained from NSRDB. Table I presents the input data from the photovoltaic system of the case study.

Table II presents the estimated parameters of the stochastic AR model for each variable. The parameters of the models shown are the order $(k)$, the coefficient $\left(a_{k}\right)$, the constant $(c)$, and the variance. The order of the model is obtained by 
applying the autocorrelation function to the historical data. Autocorrelation function results indicate the most significant lags. In this work, the smallest lag was considered to obtain only one auto-regressive term for each variable.

Table I. Input data from the photovoltaic system

\begin{tabular}{|c|c|}
\hline Model & $\begin{array}{c}\text { CS6P-270P } \\
\text { (Polycrystalline) }\end{array}$ \\
\hline Peak Power (Wp) & 270 \\
\hline Number of Panels & 80 \\
\hline Azimuth Angle ( $\left.{ }^{\circ}\right)$ & 130 \\
\hline Tilt Angle ( $\left.{ }^{\circ}\right)$ & 7 \\
\hline Performance Ratio (PR) & $75 \%$ \\
\hline Long-term Degradation & $0.75 \%$ (per year) \\
\hline Temperature Coefficient (Pmax) & $-0.41 \% /{ }^{\circ} \mathrm{C}$ \\
\hline
\end{tabular}

Table II. Estimated parameters of the AR model for each variable

\begin{tabular}{|l|c|c|c|c|c|}
\cline { 2 - 6 } \multicolumn{1}{c|}{} & $\mathbf{I}$ & $\mathbf{T}_{\mathbf{a}}$ & $\mathbf{W}$ & $\mathbf{H}$ & $\mathbf{W}_{\text {dir }}$ \\
\hline $\boldsymbol{k}$ & 9 & 1 & 1 & 1 & 12 \\
\hline $\boldsymbol{a}_{\boldsymbol{k}}$ & 0.1405 & 0.2904 & 0.1964 & 0.2888 & -0.1904 \\
\hline $\boldsymbol{c}$ & $-1.73 \mathrm{e}-5$ & $1.16 \mathrm{e}-5$ & $-7.65 \mathrm{e}-5$ & $5.31 \mathrm{e}-5$ & $7.55 \mathrm{e}-4$ \\
\hline Var. & 0.0041 & 0.0012 & 0.0091 & 0.0085 & 0.0228 \\
\hline
\end{tabular}

Fig. 2 and Fig. 3 presents the validation of the AR model with the monthly historical series. To perform this analysis, monthly synthetic series of random variables were generated for the 20 years, corresponding to the historical data period. For better visualization, a period of only 5 years is displayed in the graphs.

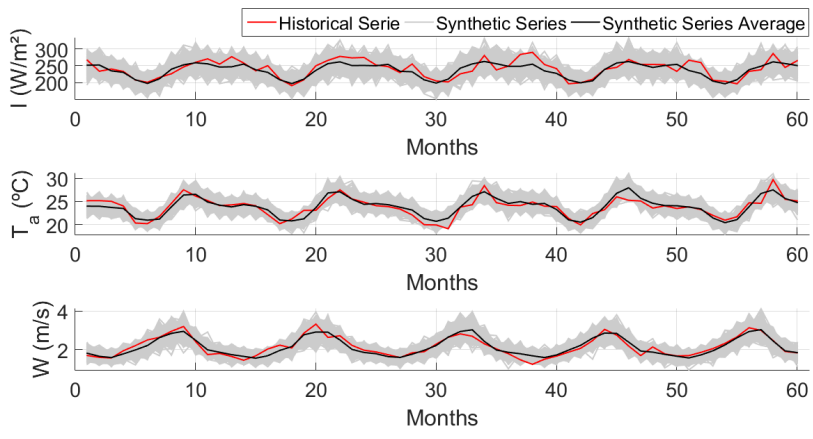

Fig. 2 - Stochastic model validation for Irradiance, Ambient Temperature, and Wind Speed

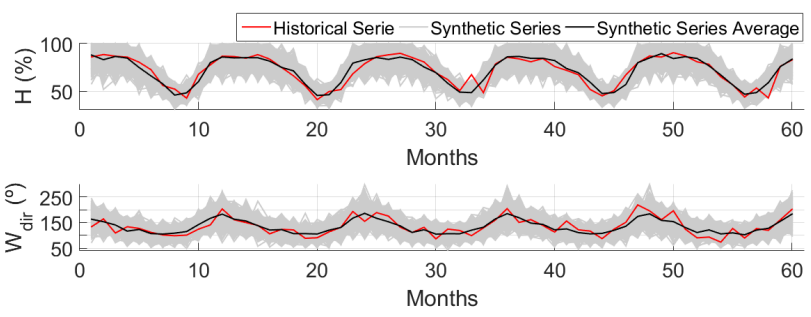

Fig. 3 - Stochastic model validation for Humidity and Wind Direction
Table III and Table IV present the statistical parameters (minimum, maximum, global mean, and standard deviation) of the monthly historical and synthetic validation series. Table IV also shows the MPE of the monthly synthetic series averages compared to the monthly historical series.

Table III. Statistical parameters of the historical series of the variables.

\begin{tabular}{|l|c|c|c|c|c|}
\cline { 2 - 6 } \multicolumn{1}{c|}{} & $\mathbf{I}\left(\mathbf{W} / \mathbf{m}^{\mathbf{2}}\right)$ & $\left.\mathbf{T}_{\mathbf{a}} \mathbf{}^{\mathbf{0}} \mathbf{C}\right)$ & $\mathbf{W}(\mathbf{m} / \mathbf{s})$ & $\mathbf{H}(\mathbf{\%})$ & $\left.\mathbf{W}_{\text {dir }} \mathbf{(}^{\mathbf{o}}\right)$ \\
\hline Min & 184.24 & 19.08 & 1.23 & 35.26 & 73.49 \\
\hline Max & 305.05 & 29.72 & 3.66 & 91.26 & 229.41 \\
\hline Mean & 236.49 & 23.79 & 2.14 & 71.42 & 134.11 \\
\hline Std & 21.39 & 1.96 & 0.48 & 14.87 & 25.90 \\
\hline
\end{tabular}

Table IV. Statistical parameters of the synthetic validation series of the variables

\begin{tabular}{|l|c|c|c|c|c|}
\cline { 2 - 6 } \multicolumn{1}{c|}{} & $\mathbf{I}\left(\mathbf{W} / \mathbf{m}^{\mathbf{2}}\right)$ & $\left.\mathbf{T}_{\mathbf{a}} \mathbf{}^{\mathbf{0}} \mathbf{C}\right)$ & $\mathbf{W}(\mathbf{m} / \mathbf{s})$ & $\mathbf{H ~ ( \% )}$ & $\left.\mathbf{W}_{\text {dir }} \mathbf{}^{\mathbf{}}\right)$ \\
\hline Min & 171.8 & 19.22 & 1.26 & 37.69 & 67.08 \\
\hline Max & 297.1 & 28.85 & 3.54 & 99.99 & 239.4 \\
\hline Mean & 236.7 & 23.75 & 2.14 & 71.46 & 134.4 \\
\hline Std & 20.83 & 1.88 & 0.46 & 14.48 & 25.56 \\
\hline MPE (\%) & 5.05 & 3.02 & 7.92 & 7.4 & 12.99 \\
\hline
\end{tabular}

It can be verified that the variables that obtained the lowest MPE values were: ambient temperature and solar irradiance, respectively. This is mainly due to the behavior of these variables and the good suitability of the AR model.

The variables with the highest MPE values were: wind direction, wind speed, and relative humidity, respectively. The bigger error in forecasting these variables is due to the high volatility of the data and a lesser adjustment to the AR model. Nevertheless, the values obtained from the global averages and standard deviations are close enough to the history for this work.

After validation of the stochastic models, the monthly synthetic series are generated for the entire project period (25 years). The statistical parameters of the series obtained in this step are presented in Table V.

Table V. Statistical parameters of the synthetic series for each variable

\begin{tabular}{|l|c|c|c|c|c|}
\cline { 2 - 6 } \multicolumn{1}{c|}{} & $\mathbf{I}\left(\mathbf{W} / \mathbf{m}^{\mathbf{2}}\right)$ & $\mathbf{T a}\left({ }^{\mathbf{0}} \mathbf{C}\right)$ & $\mathbf{W}(\mathbf{m} / \mathbf{s})$ & $\mathbf{H ~ ( \% )}$ & Wdir $\left({ }^{(}\right)$ \\
\hline Min & 170.7 & 19.12 & 1.25 & 36.76 & 65.05 \\
\hline Max & 297.9 & 28.9 & 3.58 & 99.99 & 240.9 \\
\hline Mean & 236.4 & 23.76 & 2.14 & 71.27 & 133.9 \\
\hline Std & 20.66 & 1.886 & 0.47 & 14.38 & 24.99 \\
\hline
\end{tabular}

The values of panel temperature measurements were obtained for the period from $01 / 17 / 2018$ to $04 / 16 / 2019$, corresponding to 16 months. The panel temperature was estimated through the 3 different models described by (5), (6), and (7). Fig. 4 shows the monthly measured panel temperatures $\left(\mathrm{T}_{\mathrm{PM}}\right)$ and the monthly averages of the 
synthetic panel temperature series for the three models, calculated for 16 months.

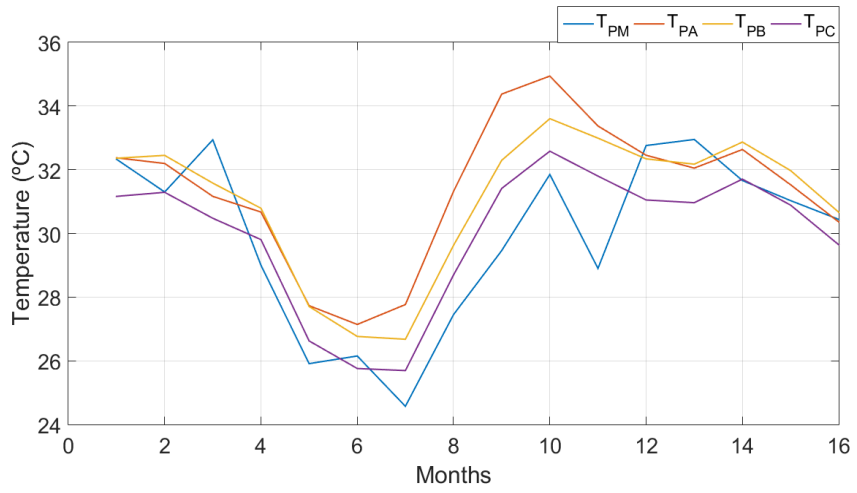

Fig. 4 - Measured and calculated panel temperature

From Fig. 4, we find that the average over the 16 months of the measured panel temperature is $29.92{ }^{\circ} \mathrm{C}$. The averages for panel temperature from models $\mathrm{A}, \mathrm{B}$, and $\mathrm{C}$ over the same period are $31.42{ }^{\circ} \mathrm{C}, 30.88{ }^{\circ} \mathrm{C}$, and $29.82{ }^{\circ} \mathrm{C}$, respectively. Therefore, model $\mathrm{C}$ is the one that has the closest mean to the average temperature measured, followed by model $\mathrm{B}$, and model $\mathrm{A}$. The average errors are $5.73 \%$, 4.67\%, and 3.77\% for Model A, Model B, and Model C, respectively. Model $\mathrm{C}$ has the smallest error among the studied models, followed by model B and model A.

Table VI presents the global averages of electrical power generated over the project lifetime for the different panel temperature models.

Table VI. Power averages provided for each panel temperature

\begin{tabular}{|l|c|c|c|}
\cline { 2 - 4 } \multicolumn{1}{c|}{} & Model A & Model B & Model C \\
\hline Average power $(\mathbf{W})$ & $3,869.6$ & $3,874.5$ & $3,892.2$ \\
\hline Std & 365.72 & 366.75 & 369.38 \\
\hline
\end{tabular}

It is interesting to note in Table VI that the standard deviations of models $\mathrm{A}, \mathrm{B}$, and $\mathrm{C}$ are influenced by the number of random variables considered. Model A, with two variables, corresponds to the smallest standard deviation. Model B, with 3 variables, exhibits a slightly larger standard deviation. And model $\mathrm{C}$, with 5 variables, has the highest standard deviation among the models. This means that the more random variables are taken into account in the panel temperature model, the more distant the average power values become.

Fig. 5 presents the averages and the standard deviations of the monthly synthetic series of generated energy for the different temperature models. The image is only represented for the first 2 years for its best visualization between the models, but the generated energy is estimated for 25 years. In Fig. 5 and Fig. $6, \mu_{\mathrm{EGA}}, \mu_{\mathrm{EGB}}$, and $\mu_{\mathrm{EGC}}$ are the averages and $\sigma_{\mathrm{EGA}}, \sigma_{\mathrm{EGB}}, \sigma_{\mathrm{EGC}}$ are the standard deviations of the monthly synthetic series of generated energy by using Model A, B, and $\mathrm{C}$.

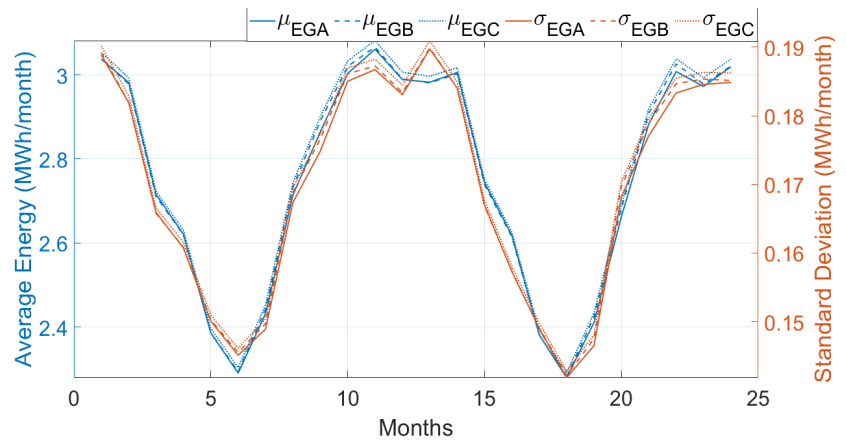

Fig. 5 - Averages and standard deviations of the synthetic series of generated energy for each temperature model

From Fig. 5, it is noted that the influence of the random variables wind speed, relative humidity, and wind direction is small on monthly energy production. The global averages of the synthetic series of energy generated over the project lifetime for each model are $2.511 \mathrm{MWh} /$ month, 2.516 $\mathrm{MWh} / \mathrm{month}$, and $2.528 \mathrm{MWh} / \mathrm{month}$, respectively.

Fig. 6 presents the average and the standard deviation of the annual energy generated over the entire project period for each temperature model. EGA is the annual generated energy calculated by Model A, EGB is the annual generated energy calculated by Model B, and EGC is the annual generated energy calculated by Model C.

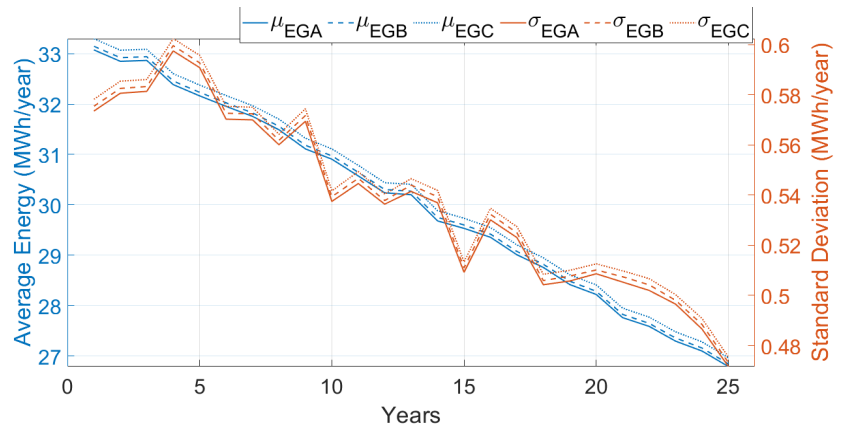

Fig. 6 - Annual average and standard deviations of energy generated for each temperature model

The decrease in generated energy found in Fig. 6 is due to the degradation of the panels' efficiency over time. The global averages of annual generated energy calculated for models A, B, and C are $30.129 \mathrm{MWh} /$ year, 30.197 $\mathrm{MWh} /$ year, and $30.332 \mathrm{MWh} /$ year. From model A to model $\mathrm{B}$ there is $0.22 \%$ increase in the yearly average energy generated over the project life. From model B to model C, there is $0.45 \%$ increase in the yearly average energy generated. From model A to model C, there is $0.67 \%$ increase in the yearly average energy generated.

\section{Conclusion and Future Work}

Validation results from both averages and errors indicated that the panel temperature model that came closest to the measurement data was Model $\mathrm{C}$, which incorporates solar irradiance, panel temperature, wind speed, relative humidity, and wind direction. Model A, which considers only the solar 
irradiance and the ambient temperature, had the worst performance, with the furthest averages from the measurements and the largest error. Model B, which considers the irradiance, the ambient temperature, and the wind speed showed intermediate performance.

Even though the panel temperature model $\mathrm{C}$ has the smallest error according to the measurements, the difference between the energy generated by the three models is very small. One of the reasons for this is that the panel temperature has less influence on photovoltaic energy production than solar irradiance. Another factor that reduces the influence of the panel temperature over the energy production is that the panel temperature variation is lesser than the solar irradiance variation throughout the year. For those reasons, the observed variations in energy generated, calculated from the different models, proved to be very close.

As a suggestion for future works, it is possible to evaluate the electricity generated by using other panel temperature models available in the literature. Due to the limitations imposed by the measurement period, the data are insufficient to obtain long-term results. Therefore, it is interesting to verify the conclusions provided from this study using a longer validation period. Validations of panel temperature models with measurement data can also be performed for different locations and technologies.

\section{Acknowledgment}

This research was supported by the Federal Institute of Goiás (IFG) and Enel Goiás. The authors would like to thank the IFG team, from campus Itumbiara, for providing us with the necessary data for this study.

\section{References}

[1] V. E. Sperling, "Hydropower in Brazil: overview of positive and negative environmental aspects", Energy Procedia, v. 18. p. $110-118,2012$.

[2] IEA, "Global Energy \& CO2 Status Report: The latest trends in energy and emissions in 2018", Available: $<$ https://www.iea.org/geco/data/ $>$.

[3] M. G. Villalva. "Photovoltaic solar energy: concepts and applications" (in Portuguese), ed. 2a 2012.

[4] M. Mitscher and R. Rüther, "Economic performance and policies for grid-connected residential solar photovoltaic systems in Brazil", Energy Policy, v. 49, p. 688-694, 2012.

[5] C. Holdermann, J. Kissel, and J. Beigel, "Distributed photovoltaic generation in Brazil: An economic viability analysis of small-scale photovoltaic systems in the residential and commercial sectors", Energy Policy, v. 49, p. 612-617, 2014.

[6] C. Lacchini and R. Rüther, "The influence of government strategies on the financial return of capital invested in PV systems located in different climatic zones in Brazil", Renewable Energy, v. 83, p. 786-798, 2015.

[7] L. F. N. Lourenço, M. B. de C. Salles, M. M. F. Gemignani, M. R. Gouvea, N. Kagan, "Time Series Modelling for Solar Irradiance Estimation in Northeast Brazil", ICREA, 2017.
[8] A. Q. Jakhrani, A. K. Othman, A. R. H. Rigit, and S. R. Samo, "Comparison of Solar Photovoltaic Module Temperature Models", World Applied Sciences Journal 14 (Special Issue of Food and Environment): 01-08, 2011.

[9] P. M. Segado, J. Carretero, and M. Sidrach-de-cardon, "Models to predict the operating temperature of different photovoltaic modules in outdoor conditions". Progress In Photovoltaics: Research And Applications, 1267-1282, 2014.

[10] R. L. Yang, G. M. Tiepolo, E. A. Tonolo, J. U. Junior, M. B. de Souza, "Photovoltaic Cell Temperature Estimation for a Grid-Connect Photovoltaic Systems in Curitiba". Smart Energy, 62, 2019.

[11] E. Pereira, J. Pinho, M. Galhardo, and W. Macêdo, "Methodology of risk analysis by Monte Carlo method applied to power generation with renewable energy". Renewable Energy, v. 69, p. 347-355, 2014.

[12] D. Pinheiro Neto, E. G. Domingues, A. Paulo Coimbra, A. T. Almeida, and W. P. Calixto, "Investment Risk Analysis for Photovoltaic Power Plant in the Free Contracting Environment", 12th International Conference on the European Energy Market, Lisboa, p.1-5. 2015b.

[13] M. Gemignani, G. J. Rostegui, and N. Kagan, "Solar radiation synthetic sequences and bidding strategies for energy auctions", 6th International Conference on Renewable Energy Research and Applications, San Diego, CA, USA, Nov. 5-8, 2017.

[14] L. F. N. Lourenço, M. B. C. Salles, M. M. F. Gemignani, M. R. Gouvea, and N. Kagan, "Time series modeling for solar irradiance estimation in northeast Brazil", 6th International Conference on Renewable Energy Research and Applications, San Diego, CA, USA, Nov. 5-8, 2017.

[15] J. M. Gordon and T. A. Reddy, "Time series analysis of daily horizontal solar radiation", Solar Energy, v. 41, n. 3, p. 215-226, 1998.

[16] G. E. P. Box and G. M. Jenkins, "Time Series Analysis, Forecasting and Control", São Francisco: Holden-Day, 1976. $575 \mathrm{p}$.

[17] H. Madsen, "Time series analysis", 1ª ed, Chapman \& Hall, 2007.

[18] L. Ljung, "System identification”, Boston: Birkhäuser, v. 1, 1998.

[19] R. J. Rossi, "Mathematical Statistics: an Introduction to Likelihood Based Inference", Wiley, 2018.

[20] M. Gemignani, G. J. Rostegui, N. Kagan, and C. F. M. Almeida, "Solar radiation synthetic series for power purchase agreements", Environmental Science and Pollution Research, 2018.

[21] A. H. A. A. Waeli, H. A. Kazem, M. T. Chaichan, K. Sopian, "The Impact of Climatic Conditions on PV/PVT Outcomes". Published in: "Photovoltaic/Thermal (PV/T) Systems". Chapter 5, 2019.

[22] G. M. Masters, "Renewable and Efficient Electric Power Systems". New Jersey: Wiley-Interscience. 2004.

[23] G. Tamizhmani, L. Ji; Y. Tang, L. Petacci, and C. Osterwald "Photovoltaic module thermal-wind performance: Long-term monitoring and model development for energy rating". NCPV and solar program review meeting. pp. 936-939, 2003.

[24] D. Pinheiro Neto, E. G. Domingues, A. P. Coimbra, A. T. Almeida, A. J. Alves, and W. P. Calixto, "Portfolio optimization of renewable energy assets: Hydro, wind, and photovoltaic energy in the regulated market in Brazil”, Energy Economics, Volume 64, Pages 238-250, 2017. 